\title{
Dynamic regulation of resource transport induces criticality in interdependent networks of excitable units
}

\author{
Yogesh S. Virkar, ${ }^{1, *}$ Juan G. Restrepo, ${ }^{2}, \dagger$ Woodrow L. Shew ${ }^{3}$ and Edward Ott ${ }^{4}$ \\ ${ }^{1}$ University of Colorado at Boulder, Boulder, CO, 80309, USA \\ ${ }^{2}$ University of Colorado at Boulder, Boulder, CO 80309, USA \\ ${ }^{3}$ University of Arkansas, Fayetteville, AR 72701, USA \\ ${ }^{4}$ University of Maryland, College Park, MD 20742, USA
}

\begin{abstract}
Various functions of a network of excitable units can be enhanced if the network is in the 'critical regime', where excitations are, on average, neither damped nor amplified. An important question is how can such networks self-organize to operate in the critical regime. Previously it was shown that regulation via resource transport on a secondary network can robustly maintain the primary network dynamics in a balanced state where activity doesn't grow or decay. Here we show that this inter-network regulation process robustly produces a power-law distribution of activity avalanches, as observed in experiments, over ranges of model parameters spanning orders of magnitude. We also show that the resource transport over the secondary network protects the system against the destabilizing effect of local variations in parameters and heterogeneity in network structure. For homogeneous networks, we derive a reduced 3-dimensional map which reproduces the behavior of the full system.
\end{abstract}

\section{INTRODUCTION}

Networks of excitable units are found in varied disciplines such as social science [1], neuroscience [2], epidemiology [3], genetics [3], etc. Various aspects of network function can be optimized when the network operates in the 'critical regime', between low and high firing rates, as in neural networks [4], or at the 'edge of chaos', between order and disorder, as in gene networks [5]. In particular, for neural networks, criticality results in potential information handling benefits [4]. A natural question receiving much interest [6-9] is what mechanisms can lead such complex and distributed systems to operate in the critical regime, which typically occurs in a very small region of parameter space. In Ref. [8] we proposed a general mechanism based on the regulation of the excitable network dynamics by a resource which enables the interactions between the excitable elements and that is transported across a secondary network. However, it was not clear if resource transport regulation is enough to produce experimental signatures of critical dynamics such as power-law distributions of avalanche sizes. In addition, the robustness of the model to parameter choices was not understood. Here we show that resource transport regulation leads to power-law distributed avalanche size distributions over model parameter ranges spanning orders of magnitude, and we validate our observations with a theoretical analysis which could serve as a basis to study more refined models of resource transport regulation. As a concrete case, we focus on the case of neural networks, where metabolic resources that facilitate the transmission of neural excitations are transported across a secondary glial network [10-13]. We emphasize, how-

\footnotetext{
*Electronic address: Yogesh.Virkar@colorado.edu

${ }_{\dagger}^{\dagger}$ Electronic address: Juan.Restrepo@colorado.edu
}

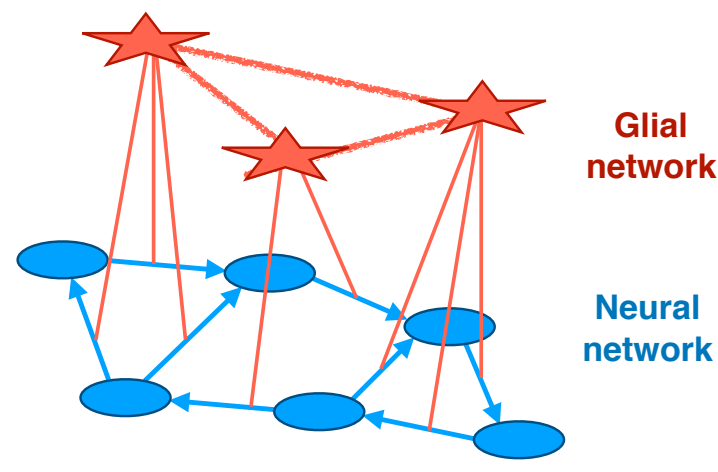

FIG. 1: Our model consists of interacting neural and glial networks. The neural network is directed and weighted while the glial network is undirected and unweighted.

ever, that our results could be applicable to other systems that operate at or near a critical point $[5,14,15]$.

\section{MODEL}

Following [8], we consider a network model with two interdependent networks: a weighted directed neural network, and an unweighted undirected glial network which transports and regulates the supply of resources needed for the functioning of the neural network (see Fig. 1).

Neural network dynamics: The neural network consists of $N$ excitable nodes that represent neurons, labeled $n=$ $1,2, \ldots, N$, and $M$ directed edges (each corresponding to a synapse) and labeled $\eta=1,2, \ldots, M$. We also indicate a synapse pointing from node $m$ to node $n$ by $\eta_{(n, m)}$. At each discrete time step, $t$, neuron $n$ is in either the quiescent state $\left(s_{n}^{t}=0\right)$ or the active state $\left(s_{n}^{t}=1\right)$. We define $W^{t}$ as the $N \times N$ adjacency matrix whose entry $W_{n m}^{t}$ denotes the weight of the synapse on the edge from 
neuron $m$ to neuron $n$ at time $t$. The state of neuron $n, s_{n}^{t}$, is updated probabilistically based on the sum of its synaptic input from active presynaptic neurons in the previous time step,

$$
s_{n}^{t+1}=\left\{\begin{array}{l}
1 \text { with probability } \sigma\left(\sum_{m=1}^{N} W_{n m}^{t} s_{m}^{t}+\mu\right) \\
0 \text { otherwise } .
\end{array}\right.
$$

As in Refs. [8, 16], the model transfer function probability $\sigma$ is piecewise linear; $\sigma(x)=0$ for $x \leq 0, \sigma(x)=x$ for $0<x<1$, and $\sigma(x)=1$ for $x \geq 1$, and $\mu=1 / 15000$ is a small external input that allows the system to avoid getting trapped in the absorbing state $s_{n}=0$ for all $n$.

At time $t$ each synapse $\eta$ is assumed to have a supply $R_{\eta}^{t}$ of a metabolic resource, some of which is consumed every time the presynaptic neuron, $m(\eta)$, fires. While in this paper we do not focus on a particular resource, we note that $R$ could represent various metabolites that are transported diffusively among the glial cells, such as glucose and lactate [17]. Reflecting the increasing synaptic firing ability with increasing resource, we assume that the weight $W_{n m}^{t}$ in synapse $\eta_{(n, m)}$ from neuron $m$ to neuron $n$ is proportional to the amount of resource in the synapse, $W_{n m}^{t}=w_{n m} R_{\eta(n, m)}^{t}$ [8]. Finally, for simplicity, we consider only excitatory neurons and assume that there is no learning (these were considered in [8]). Thus, synaptic weight changes are caused only by the dynamics of resource transport.

The second network of our model, the unweighted and undirected glial network, consists of $T$ glial cells labeled $i=1,2, \ldots, T$. Each glial cell $i$ holds an amount of resource $R_{i}^{t}$ at time step $t$. Resources diffuse between the glial cells that are connected to each other. We define a $T \times T$ symmetric glial adjacency matrix $U$ such that entry $U_{i j}=1$ if glial cell $j$ is connected to glial cell $i$ and $U_{i j}=0$ otherwise. Each glial cell serves a set of synapses by supplying resource to them. Hence we define a $T \times M$ matrix $G$ with entries $G_{i \eta}=1$ if glial cell $i$ serves synapse $\eta$ and $G_{i \eta}=0$ otherwise.

Resource-transport dynamics: Resource diffuses between glia through their connection network (characterized by the adjacency matrix $U$ ) and between glia and the synapses they serve (via the glial-neural connection network characterized by the adjacency matrix $G$ ). Our model for the evolution of the amount of resource $R_{i}^{t}$ at glial cell $i$ and the amount of resource $R_{\eta}^{t}$ at synapse $\eta$ is

$$
\begin{aligned}
R_{i}^{t+1} & =R_{i}^{t}+C_{1}+D_{G} \sum_{j=1}^{T} U_{i j}\left(R_{j}^{t}-R_{i}^{t}\right) \\
& +D_{S} \sum_{\eta=1}^{M} G_{i \eta}\left(R_{\eta}^{t}-R_{i}^{t}\right), \\
R_{\eta}^{t+1}= & R_{\eta}^{t}+D_{S}\left(R_{i(\eta)}^{t}-R_{\eta}^{t}\right)-C_{2} s_{m(\eta)}^{t},
\end{aligned}
$$

where $D_{G}$ is the rate of diffusion between glial cells, and $D_{S}$ is the rate of diffusion between glia and synapses. Moreover, we enforce $R_{\eta} \geq 0$, i.e., if Eq. (3) yields $R_{\eta}^{t+1}<0$, then we replace it by 0 . The model parameter $C_{1}$ on the right hand side of Eq. (2) denotes the amount of resource added to each glial cell at each time step (e.g., supplied by capillary blood vessels). For simplicity, we assume first that each glial cell has the same $C_{1}$ (the effect of heterogeneous values of $C_{1}$ will be discussed later). The last two terms in Eq. (2) are the amount of resource transported to glial cell $i$, respectively, from its neighboring glial cells and from the synapses that it serves.

The term proportional to $D_{S}$ in Eq. (3) denotes the amount of resource gained (if $R_{i(\eta)}^{t}>R_{\eta}^{t}$ ) or lost (if $R_{i(\eta)}^{t}<R_{\eta}^{t}$ ) from glial cell $i(\eta)$ that serves synapse $\eta$. If the presynaptic neuron $m(\eta)$ fires at time step $t$ $\left(s_{m(\eta)}^{t}=1\right)$, then synapse $\eta$ consumes an amount of resource $C_{2}$, thus decreasing the resource at synapse $\eta$ by this amount.

\section{NUMERICAL EXPERIMENTS}

We now describe and present the results of numerical experiments on our model, Eqs. (1)-(3). Our main goal is to show that resource transport dynamics robustly regulates the operation of the neural network in the critical regime. In the neural model used here, the critical regime is characterized by the largest eigenvalue of the neural synapse matrix $W^{t}, \lambda^{t}$, being one $[8,16]$. Therefore we will consider $\lambda^{t} \approx 1$ as one criterion for criticality. However, a more practical definition of criticality, applicable more generally [4], is a power-law distribution of the sizes of activity bursts, or neuronal avalanches. We will also verify that the model robustly produces power-law distributed neuronal avalanches.

In our numerical experiments, we consider an ErdösRényi network structure for both the neural and glial networks. The neural network is described using an $N \times$ $N$ adjacency matrix, $W$, such that with probability $p$ we have an entry $w_{n m} \neq 0$ that represents a link from node $m$ to node $n$. At time $t=0$, we set the resource at each synapse $\eta, R_{\eta}^{0}=1$, and draw $w_{n m}$ from a uniform distribution over $[0, \bar{w}]$. By choosing the value of $\bar{w}$, we can set the initial largest eigenvalue of $W^{t}$, i.e., $\lambda^{0}$, to a desired value, and test whether the subsequent evolution of the model results in $\lambda^{t} \rightarrow 1$.

The glial network is undirected and unweighted and its adjacency matrix is given by a $T \times T$ matrix, $U$, such that with probability $q$ we have an entry $U_{i j}=U_{j i}=1$ that represents an undirected link between nodes $i$ and $j$. Motivated by experiments [18] showing $T \sim N$, for specificity, we take the number of glia and neurons to be equal, $T=N$. Consistent with this, and the additional experimental finding that all incoming synapses of a given neuron are served by the same glial cell [19], we further assume that each glial cell serves a unique neuron. We set the initial resource for each glial cell to be 

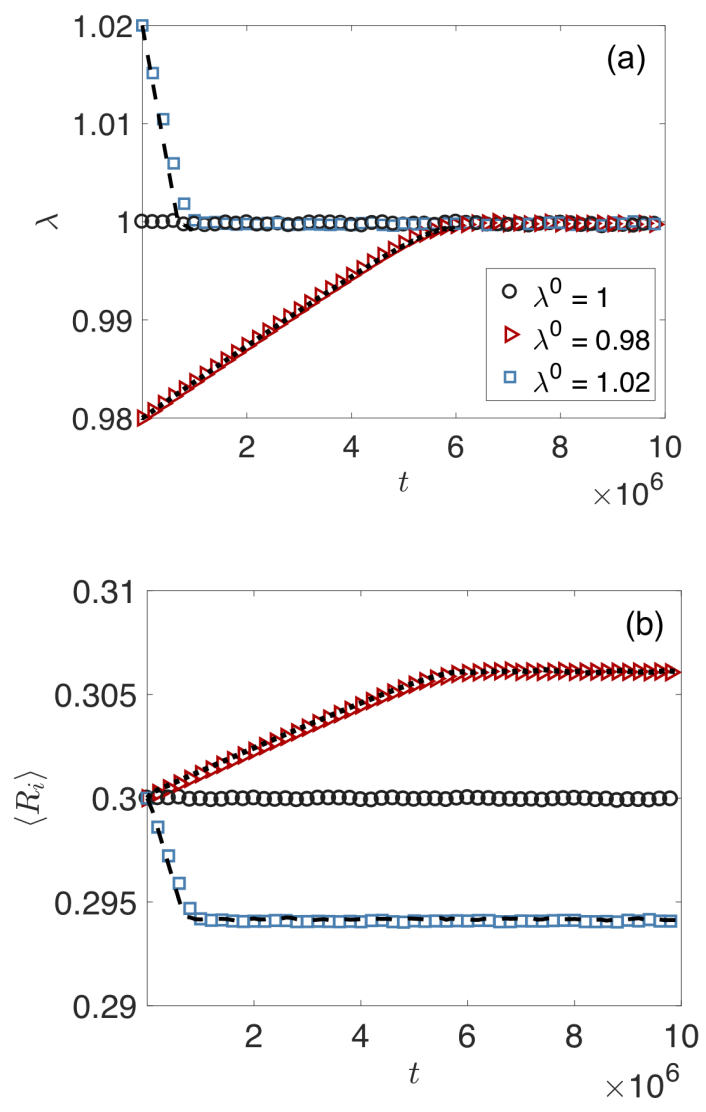

FIG. 2: (a) Largest eigenvalue, $\lambda^{t}$, of the neural network adjacency matrix for three initial conditions $\lambda^{0}=1,0.98,1.02$ (black circles, red triangles, and blue squares, respectively) as a function of time. (b) Average glial resource $R^{t}=\sum_{i} R_{i}^{t} / N$ as a function of time for the same initial conditions. In both panels, the dotted (subcritical case) and the dashed (supercritical case) lines show the predictions from the 3-D map with noise [Eqs. (9), (11),(13)].

equal to an uniform value, $R_{i}^{0}=r$ (note that $R_{\eta}^{0}$ may be different from $R_{i}^{0}$ ). For all numerical experiments we use the values $T=N=1000, p=q=0.05$, and assume, for simplicity, that the entries of matrices $U$ and $W$ are independent of each other. Unless mentioned otherwise, the parameters for resource-transport dynamics are set as $D_{G}=D_{S}=5 \times 10^{-5}, C_{1}=6 \times 10^{-8}$, and $C_{2}=10^{-8}$.

In the first experiment, we show that starting with different initial conditions $\lambda^{0}=1,0.98,1.02$, the resourcetransport dynamics causes the system to self-organize to the critical state corresponding $\lambda^{t}=1$ after a transient period. In Fig. 2 (a) we show $\lambda^{t}$ for the three different initial conditions $\lambda^{0}=1,0.98,1.02$ (black circles, red triangles, and blue squares, respectively). In the three cases, $\lambda^{t}$ approaches and subsequently remains close to 1 (this will be quantified in Fig. 4). In Fig. 2 (b) we show that the average glial resource, $R^{t}=\sum_{i} R_{i}^{t} / N$, reaches a steady state in all three cases.

As discussed above, we are interested in whether the dynamics of the neuronal network reproduces experimen- tal signatures of critical behavior, in particular power-law distributed avalanches of activity. To do this, following [16], we define a measure of activity, $S(t)=\sum_{m} s_{m}^{t} / N$, and define an avalanche as the excursion of activity $S^{t}$ above a threshold $S^{*}$, i.e., $S^{t}<S^{*}$ for $t<t_{1}, t>t_{2}$ and $S^{t} \geq S^{*}$ for $t_{1} \leq t \leq t_{2}$ ). We define the size $L$ of the avalanche as $L=N \sum_{t=t_{1}}^{t_{2}} S^{t}$, the number of spikes (excitations) over a single excursion.

To investigate the robustness of our model to changes in parameters, we fix $D=D_{G}=D_{S}=5 \times 10^{-5}$ and vary $C_{1}$ and $C_{2}$ logarithmically roughly from $10^{-8}$ to $10^{-2}$ keeping the ratio $C_{2} / C_{1}=1 / 6$ constant. Using the threshold $S^{*}=0.15$, we calculate avalanche size distributions, $P(L)$, for each parameter setting. We then fit a power-law model using standard techniques [20] based on maximum-likelihood estimation (MLE) and a hypothesis test that generates a $p$-value using the KolmogorovSmirnov (KS) statistic. Since we have finite-size effects, in addition to the lower size $L_{\min }$ cutoff used in [20], we introduce an upper cutoff $L_{\max }$, i.e., we test the plausibility of a power-law model where we condition on avalanche sizes in a range $\left[L_{\min }, L_{\max }\right][21,22]$. We accept as plausible power laws only those distributions for which this range spans at least three decades.

In Fig. 3 we show size distributions $P(L)$ for various values of $C_{1}$. The blue curves indicate a plausible powerlaw fit (under $10 \%$ level of significance) with $P(L) \propto L^{\gamma}$ such that $\gamma \approx-3 / 2$ (exponents range from -1.42 to -1.49 ), and the red curves a rejected power-law fit. We find that there is a large range of values of the parameters (small values of $C_{1}$ and $C_{2}$ for which the system results in power-law distributed avalanches, but that for some parameter choices (larger values of $C_{1}$ and $C_{2}$ ) the distribu-

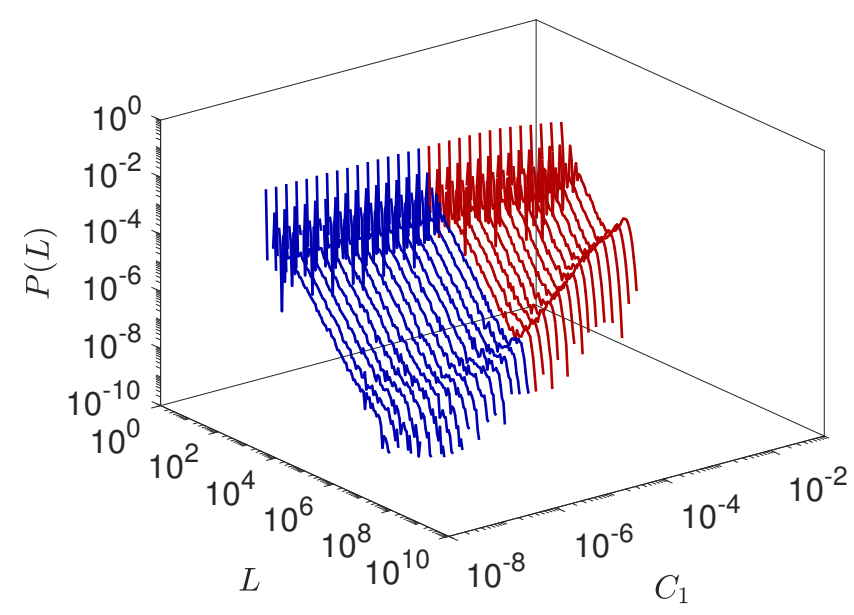

FIG. 3: Size distributions $P(L)$ for various values of $C_{1}$. Blue curves indicate plausible power-law fits (under $10 \%$ level of significance) with $P(L) \propto L^{\gamma}$ such that $\gamma \approx-3 / 2$ and the red curves indicate rejected power-law fits. 
tion of avalanche sizes no longer satisfies our KolmogorovSmirnov power-law test.

\section{3-DIMENSIONAL MAP}

In order to gain an understanding of the mechanisms that lead to the critical regime and to determine conditions on the model parameters that result in critical behavior, we make the following assumptions: (i) the neural network is large, uncorrelated and homogeneous so that the Perron-Frobenius eigenvalue of the matrix with entries $W_{n m}=w_{n m} R_{\eta(n, m)}$ is well approximated by its average row sum, (ii) the intrinsic synapse weights $w_{n m}$ are uncorrelated with $R_{n m}$ or have a narrow distribution around their average $\langle w\rangle$ so that $\sum_{n, m} w_{n m} R_{\eta(n, m)}^{t} \approx$ $\langle w\rangle \sum_{n, m} R_{\eta(n, m)}^{t}$, and (iii) the glial cells all serve the same number of synapses $k$ (or the distribution of the number of synapses served is narrow). While some of these assumptions could be relaxed and the theory generalized, we leave these considerations for future work.

First, we define the average amount of resource per glial cell at time $t$ :

$$
R^{t}=\frac{1}{T} \sum_{i} R_{i}^{t}
$$

Averaging Eq. (2) over glial cells, we obtain

$R^{t+1}=R^{t}+C_{1}+\frac{D_{S}}{T} \sum_{\eta=1}^{M} R_{\eta}^{t} \sum_{i=1}^{T} G_{i \eta}-\frac{D_{S}}{T} \sum_{i=1}^{T} R_{i}^{t} \sum_{\eta=1}^{M} G_{i \eta}$.

From the assumption that each glial cell serves $k$ synapses, we have $\sum_{\eta=1}^{M} G_{i \eta}=k$. Furthermore, since each synapse is served by a unique glial cell, $\sum_{i=1}^{T} G_{i \eta}=$ 1 , and we obtain

$$
R^{t+1}=R^{t}+C_{1}+\frac{D_{S}}{T} \sum_{\eta=1}^{M} R_{\eta}^{t}-k D_{S} R^{t}
$$

The term $\sum_{\eta=1}^{M} R_{\eta}$ is the total amount of resource in the synapses. From the assumption that the fixed synapse weights $w_{n m}$ are uncorrelated with $R_{n m}$ (or that their distribution is sufficiently narrow), the total resource in the synapses can be related to the sum of all entries $W_{n m}^{t}=w_{n m} R_{n m}^{t}$ of the synapse weight matrix

$$
\sum_{\eta=1}^{M} R_{\eta}^{t}=\frac{1}{\langle w\rangle} \sum_{n, m} W_{n m}^{t}
$$

For large homogeneous, uncorrelated networks, the average row sum is an excellent approximation to the PerronFrobenius eigenvalue, and so

$$
\sum_{\eta=1}^{M} R_{\eta}^{t} \approx \frac{N}{\langle w\rangle} \lambda^{t}
$$

and Eq. (6) becomes, using $N=T$ as discussed before,

$$
R^{t+1}=R^{t}+C_{1}+\frac{D_{S}}{\langle w\rangle} \lambda^{t}-k D_{S} R^{t}
$$

Summing Eq. (3) over $\eta$ and multiplying by $\langle w\rangle / N$ we get

$$
\lambda^{t+1}=\lambda^{t}+\frac{D_{S}\langle w\rangle}{N} \sum_{\eta=1}^{M} R_{i(\eta)}^{t}-D_{S} \lambda^{t}-\frac{C_{2}\langle w\rangle}{N} \sum_{\eta=1}^{M} s_{m(\eta)} .
$$

Since there is a single glial cell serving each synapse, and each synapse serves $k$ glial cells, $\sum_{\eta=1}^{M} R_{i(\eta)}^{t}=$ $\sum_{i=1}^{T} k R_{i}^{t}=T k R^{t}=N k R^{t}$. In addition, since each glial cell serves all the $k$ synapses of a single neuron, $\sum_{\eta=1}^{M} s_{m(\eta)}=k \sum_{m=1} s_{m}^{t}=k N S^{t}$. So we obtain

$$
\lambda^{t+1}=\lambda^{t}+D_{S}\langle w\rangle k R^{t}-D_{S} \lambda^{t}-C_{2}\langle w\rangle k S^{t} .
$$

The mean field equations (9), (11) need to be closed with an equation for the evolution of the average activity, $S^{t}$, which is a stochastic variable determined by Eq. (1). In order to obtain a tractable map, we model $S^{t}$ in two different ways: in the first one, we neglect stochastic effects and use the deterministic approximation:

$$
S^{t+1}=\lambda^{t} S^{t}
$$

This approximation is based on the fact that, except for values of $S$ close to 0 or 1 , the expectation of $S^{t+1}$ calculated from Eq. (1) is $\lambda^{t} S^{t}$. This approximation neglects the nonlinear effects that keep $S^{t}$ below 1, and thus should be interpreted only as a guide to determine the fixed points and their stability in the limit of vanishing stochastic effects [i.e., when the expected number of terms in the sum in Eq. (1) is large]. We refer to Eqs. (9), (11) and (12) as the 3-D map without noise. A more realistic model for $S^{t}$ includes a stochastic noise term and a mechanism to enforce $0 \leq S^{t} \leq 1$ :

$$
S^{t+1}=\max \left(0, \min \left(1, \lambda^{t} S^{t}+r^{t}+\mu^{t}\right)\right) .
$$

Here, $r^{t}$ is a Gaussian noise term with zero mean and standard deviation $\sqrt{S^{t}\left(1-S^{t}\right) / N}$, as estimated in [16], while $\mu^{t}$ represents an external stimulus taken to be $1 / N$ with probability $\zeta$ and 0 otherwise, introduced to prevent $S^{t}$ from decaying to zero. Effectively, this stimulus excites one neuron every time step with probability $\zeta$. We refer to Eqs. (9), (11) and (13) as the 3-D map with noise. This variant of the map is useful to predict the evolution of the macroscopic variable $R^{t}$ of the full model. As an example, in Fig. 2 we show with dotted and dashed lines the predictions of the evolution of $\lambda^{t}$ and the average glial resource $R^{t}$ obtained from the 3-D map with noise. The predictions agree very well with direct simulations of the full model.

The simplicity of the 3-D map without noise allows us to derive parameter constraints that must be satisfied for 
a stable critical state. In particular, the system of Eqs. (9), (11), (12) has the fixed point

$$
\bar{\lambda}=1, \quad \bar{S}=\frac{C_{1}}{k C_{2}}, \quad \bar{R}=\frac{C_{1}}{k D}+\frac{1}{k\langle w\rangle} .
$$

The critical state $\lambda=1$ is a fixed point of the deterministic map. Its stability is determined by whether the eigenvalues of the Jacobian of the map (9), (11), (12) evaluated at the fixed point (14) are inside the complex unit circle. Applying the Routh-Hurwitz criterion, we find that the fixed point is stable (which we interpret as robustness of the critical state) if

$$
\begin{aligned}
& D k-\frac{2}{3}<0, \\
& \frac{1}{k D}-\frac{1+k}{C_{1} k\langle w\rangle}-\frac{3}{4}<0, \\
& \frac{C_{1} k D\langle w\rangle}{8}-\frac{C_{1}\langle w\rangle}{4}+\frac{D k}{2}+\frac{D}{2}-1<0, \\
& C_{1}^{2} D^{2} k^{2}\langle w\rangle-2 C_{1}^{2} D k\langle w\rangle+C_{1}^{2}\langle w\rangle \\
& +C_{1} D^{2} k^{2}+C_{1} D^{2} k-C_{1} D<0 .
\end{aligned}
$$

In addition, since $0 \leq \bar{S} \leq 1$, we have the additional inequality $C_{1} /\left(k C_{2}\right)<1$, which represents the constraint that the amount of resource supplied to glial cells per time step can not exceed the amount that can be consumed at the synapses.

To demonstrate the usefulness of these inequalities, we verify which of the curves in Figure 3 correspond to parameters which satisfy these inequalities. Parameters that satisfy (don't satisfy) the inequalities approximately correspond to distributions which follow (don't follow) a power-law. To illustrate this, we plot in Fig. 4 the quantity $\sigma_{\lambda}=\sqrt{\left\langle\left(\lambda^{t}-1\right)^{2}\right\rangle_{t}}$, which measures the deviation of the system from $\lambda=1$, as a function of $C_{1}$ (we take

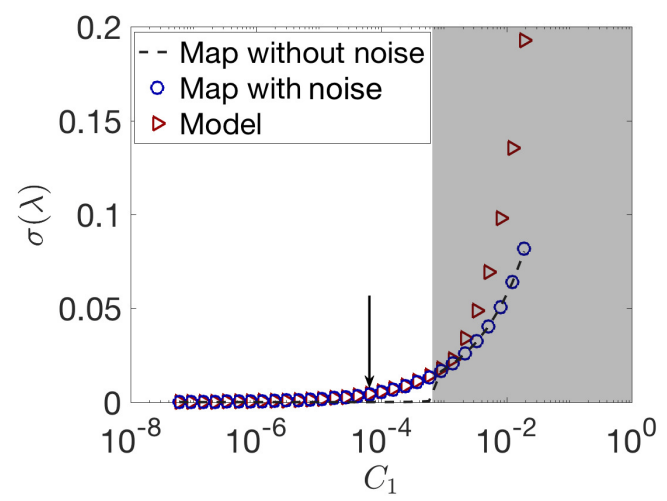

FIG. 4: Root mean squared deviation of $\lambda^{t}$ from $1, \sigma_{\lambda}=$ $\sqrt{\left\langle\left(\lambda^{t}-1\right)^{2}\right\rangle_{t}}$ as a function of $C_{1}$. The shaded grey region indicates parameter values for which $\bar{\lambda}=1$ is linearly unstable. Values of $C_{1}$ to the left of the arrow yield avalanche size distributions that have plausible power-law fits with exponent close to $-3 / 2$.
$C_{2}=C_{1} / 6, D=5 \times 10^{-5}$, and $k=N p$ ). The red triangles correspond to simulations of the full model, the blue circles to the 3-D map with noise, and the dashed line to the 3-D map without noise. The shaded grey region indicates parameter values for which the linear stability analysis predicts the fixed point $\bar{\lambda}=1$ to be unstable. We observe that the 3-D map with noise captures the deviations from $\lambda=1$ very well until these become large slightly past the onset of instability, i.e., approximately when $\sigma_{\lambda} \approx 0.025$. The 3-D map without noise, neglecting fluctuations, fails to capture the small deviations from $\lambda=1$ that occur before the onset of instability. To relate these findings with the distribution of avalanche sizes, we indicate with an arrow the value of $C_{1}^{*}$ such that values $C_{1}<C_{1}^{*}$ yield avalanche size distributions that have plausible power-law fits with exponent close to $-3 / 2$ (the same information that was used to color the curves in Fig. 3). The map without noise thus predicts roughly the onset of instability and, correspondingly, of avalanche size distributions that are not power-law distributed.

So far, our results have been independent of resource transport in the glial network. The resource supply and consumption could be understood as a local homeostatic mechanism analogous to those discussed in Refs. [23, 24] and references therein. However, in the next numerical experiment we show that, when there are heterogeneities (in the network structure, in the supply and consumption rates $C_{1}$ and $C_{2}$, etc), the diffusion of resources between glial cells can compensate for these effects and prevent destabilization of the critical state. We consider the particular case of heterogeneous source rates, where now each glial cell $i$ has its own $C_{1}^{i}$. As an example, we draw the $C_{1}^{i}$ from a Gaussian distribution with mean $C_{1}$ and standard deviation $2.6 \times 10^{-7}$, so that approximately $5 \%$ of them do not satisfy the inequality $C_{1}^{i}<k C_{2}$. In the absence of resource transport, resource accumulates in these glial cells and the associated synapses, bringing the network to the supercritical state, $\lambda^{t}>1$. However, when resource is allowed to diffuse, the critical state $\lambda^{t} \approx 1$ is maintained. This is shown in Fig. 5, where we plot $\lambda^{t}$ as a function of time with $D_{G}=0$ (red triangles) and $D_{G}=D_{S}>0$ (blue circles). We also note that in addition to stabilizing the critical state against parameter heterogeneities, network diffusion can stabilize the critical state in the presence of learning [8].

\section{DISCUSSION}

To summarize, we have found that resource-transport dynamics can stabilize the dynamics of excitable units so that they operate at a critical state characterized by experimentally-observable power-law distributed avalanche sizes. Using a reduced 3-dimensional map, we showed that for a large range of parameters the system self-organizes to a critical state that is characterized by power-law distributed avalanche sizes with an exponent 


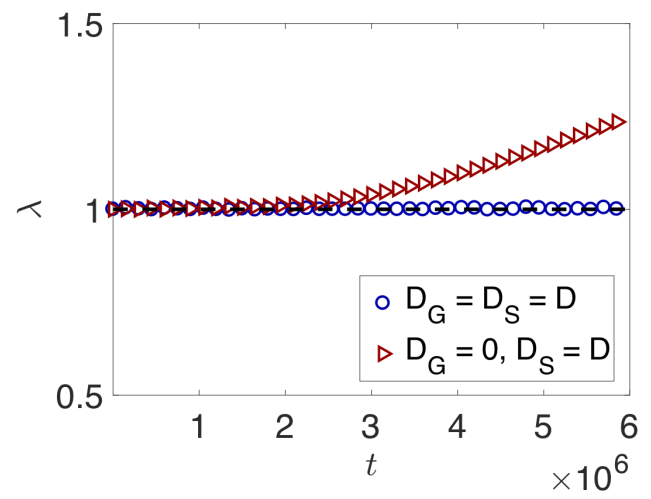

FIG. 5: Using heterogeneous source rates, $\lambda$ remains close to 1 when there is diffusion amongst glia, i.e., $D_{G}=D$ (blue circles), and grows when resource transport amongst the glial cells is absent, i.e. $D_{G}=0$. The dashed line shows $\lambda=1$ for reference. value near the characteristic $-3 / 2$ exponent found in various experimental studies. We found that resource transport dynamics protects the system against the destabilizing effects of parameter heterogeneity. While our theoretical analysis is based on the assumption of a homogeneous network, it could potentially be generalized to account for heterogeneous or spatial network structure. Although we presented our model in the context of neuronal networks, our results could be applicable to other networks of excitable elements whose interaction efficacy depends on the availability of a shared resource.
[1] S. Pei, S. Tang, and Z. Zheng. PLoS ONE, 10, e0124848 (2015).

[2] D. B. Larremore, W. L. Shew, and J. G. Restrepo. Phys. Rev. Lett., 106, 058101 (2011).

[3] M. E. J. Newmann. SIAM Rev., 45, 167 (2003).

[4] W. L. Shew and D. Plenz. Neuroscientist, 19, 88 (2013).

[5] S. A. Kauffman. J. Theo. Biol., 22, 437 (1969).

[6] Anna Levina, J Michael Herrmann, and Theo Geisel. Nature physics, 3(12):857, 2007.

[7] Ludmila Brochini, Ariadne de Andrade Costa, Miguel Abadi, Antônio C Roque, Jorge Stolfi, and Osame Kinouchi. Scientific reports, 6:35831, 2016.

[8] Y. S. Virkar, W. L. Shew, J. G. Restrepo, and E. Ott. Phys. Rev. E, 94, 042310 (2016).

[9] Osame Kinouchi, Ludmila Brochini, Ariadne A Costa, João Guilherme Ferreira Campos, and Mauro Copelli. Scientific reports, 9(1):3874, 2019.

[10] L. Roux D. Holcman C. Giaume, A Koulakoff and N. Rouach. Nature Reviews Neuroscience, 11(2):87-99

[11] D. H. Woo R. D. Fields and P. J. Basser. Neuron, $86(2): 374-386$

[12] O. Bozdagi G. W. Huntley R. H. Walker P. J. Magistretti A. Suzuki, S. A. Stern and C. M. Alberini. Cell, 144(5):810-823

[13] C Giaume, A. Koulakoff, L. Roux, D. Holcman, and N. Rouach. Nat. Rev. Neurosci., 11, 87 (2010).
[14] Manfred G Kitzbichler, Marie L Smith, Søren R Christensen, and Ed Bullmore. PLoS computational biology, 5, 2009.

[15] Bhaskar Krishnamachari, Stephen B Wicker, and Ramon Bejar. In GLOBECOM'01. IEEE Global Telecommunications Conference (Cat. No. 01CH37270), 2001.

[16] D. B. Larremore, W. L. Shew, E. Ott, F. Sorrentino, and J. G. Restrepo. Phys. Rev. Lett., 112, 138103 (2014).

[17] N. Rouach, A. Koulakoff, V. Abudara, K. Willecke, and C. Giaume. Science, 322, 1551 (2008).

[18] F. A. C. Azevedo et al. J. Comp. Neurol., 513, 532 (2009).

[19] M. M. Halassa, T. Fellin, H. Takano, J-H. Dong, and P. G. Haydon. J. Neurosci., 27, 6473 (2007).

[20] A. Clauset, C. R. Shalizi, and M. E. J. Newmann. SIAM Rev., 51, 661 (2009).

[21] D. Langlois, D. Cousineau, and J.-P. Thivierge. Physical Review E, 89(1):012709, 2014.

[22] J. Pobst Y. Karimipanah N. C. Wright W. L. Shew, W. P. Clawson and R. Wessel. Nature Physics, 11(8):659-663

[23] J. Zierenberg, J. Wilting, and V. Priesemann. Physical Review X, 8, 031018 (2018).

[24] F. Y. Kalle Kossio, S. Goedeke, B. van den Akker, B. Ibarz, and R.-M. Memmesheimer. Physical Review Letters, 121, 058301 (2018). 